\title{
Object Interaction in Object-Oriented Deductive Conceptual Models
}

\author{
Carme Quer \\ Antoni Olivé \\ Universitat Politecnica de Calalunya \\ Facultat d Tnformatica de Barcelona \\ Pau Gargallo 5 \\ 08028 Barcclona - Catalonia \\ ( cquer l olive) (a) lsi.upc.es
}

\begin{abstract}
We present the main components of an object-oriented deductive approach to conceptual modelling of inlormation systems. This approach does not model object interaction explicilly. Ilowever interaction anong objects can be clerived by means of a formal procedure that we outline.

Based on our results, we discuss whether explicit object interaction is a desirable feature of conceptual models.
\end{abstract}

\section{Introduction}

The purpose of this paper is threelold:

a) To present the main components of an object-oriented deductive approach to conceptual modelling of information systems.

b) To outline a lormal procedure for the derivation of object interactions in an objectoriented deductive conceptual model.

c) To show that most difficulties in the modelling of the dynamic aspect with current object-oriented methods arise because they try to model explicilly the interaction anong objects, which is not necessary from a conceptual point of view.

Deductive conceplual molels (DCMs) model an Information Base (IB) [JaR84] by means of predicates. Predicates may be base, corresponding to external events, derived, corresponding to entities, attributes and generated events, or constraints, corresponding to the integrity constraints that the IB must satisly al any time. Deduction rules define derived facts in terms of base and/or other derived facis [Oli89,MSS92]. However, DCMs are flat, in the sense that they lack a structuring mechanism of the (usually large) set of predicates.

We provide here a combination of the deductive and object-oriented approaches, by which we group the IB predicates using the concept of object. We do not intend here to present specific language features, but the CIAM language [GKB82] could be a materiadization of our approach. The object-oricnted deductive conceptual models (ODCMs) thus oblained still have all advantages over the operational models [Oli86], while gaining in modularization. 
ODCMs do not model object interaction explicilly. Any deduction rule may refer to any event, object or attribute. However, it is interesting to derive the object interactions implied by a given ODCM. This may be useful as a validation tool and as a preliminary step towards an ulterior object-oriented design. To this end, we have developed a fornal procedure that produces the object interaction patterns that may result from an ODCM.

This procedure shows that object interaction cmbodies some design decisions and that several distinct object intcraction patterns are possible. This might be the reason why the specification of the interaction among objects is a difficult issue in object-oriented methods, as can be seen from the greal diversily of approaches taken by current methods [dCP92]. Our findings suggest that explicit object interaction should perhaps not be defined at the conceptual level.

The paper is structured in 6 sections. Next section presents the ODCMs, including an example that will be used throughout the paper. Section 3 briefly reviews the concepts of internal event and internal events model. Section 4 describes a procedure that uses this model to derive object interactions in an ODCM. Section 5 then discusses our approach with respect to the couventional object-oriented approaches. Finally, Section 6 gives the conclusions and points out luture work.

\section{Object-Oriented Deductive Conceptual Models}

In this section we characterise the main features of an ODCM in a first-order logic framework. We try to concentrate on the main issues and, therefore, we refrain from explaining unnecessary deails. We will use the ODCM example given in Figures 1 and 2 throughout the paper.

An ODCM consists of a set $E$ of external event classes and a set $O$ of object classes.

\subsection{External Event Classes}

External events correspond to the inputs received by the Information System from the environment. The definition of an exterual event chass consists of:

- The attributes of the events. We distinguish between altributes provided by the environment and derived attributes, i.e. those attributes whose value must be computed when the event occurs. We assume that each event has an implicit time atribute that gives, in a suitable time unit (such as seconds), the instant when the event occurs. For each derived attribute, we deline one or more deduction rules.

- The constraints that the events must sitisfy to be valid. For each constraint we define a deduction rule. These constraints must be verified when the events occur.

Deduction rules have the form: $p(X) \leftarrow \Phi$, where $X$ is a vector of variables, $\Phi$ a first order formula, and where all free variables are universally quantified. Note that we do not restrict deduction rules to Horn clauses (with negation) at this level. 
Additional details will be explained using the example of Figure 1. We assume that each event has a unique identifier, which is provided automatically by the system. A fact such as sale $(z, 1)$ is true if a salc event with identifier $z$ occurred at time $t$. A fact such as qty $(z: q)$ is true, where $z$ is the identifier of a sale event, ir the qly attribute of $z$ has value $q$. Note the difference in notation: $\operatorname{salc}(z, 1)$ denoles the existence of a sale event $z$ at time $t$, while $q t y(z: q)$ denotes $q$ as the value of the qty aturibute of the sale event $z$.

Instances of new_prod event class have two altribules: prodno (with domain integer), which is provided by the environment, and prod (with domain the set of identifiers of objects of class PRODUCT), which is derived. The deduction rule states how the prod attribute is defined: in this case, the product identifier is just the prodno value. To save space, we have omitted similar dedaction rules in the rest of Figure 1.

There is a constraint associated with event class new_prod. The name of the constraint is prod_exists, which is a predicate name. A lact prod_exists(p,t) is true, meaning that, the constraint is violated, if there exists an event new_prod $(z, 1)$, such that its prod attribute has value $p$, and $p$ is a product at time $1-1$. Note that, as we explain below, product(p,t-1) is true if $p$ is an object of class PRODUCT at time $1-1$. It is assumed that the external events that violate a constraint (a new_prod in this example) will be rejected. We also omit similar constraint rules in the rest of Figure 1.

external events

event new_prod (prodno: integer; derived prod: PRODUCT).

$\operatorname{prod}\left(Z: \mathrm{P}^{\mathrm{P}}\right) \leftarrow \operatorname{prodno}(\mathrm{Z}: \mathrm{P})$.

constraints

$\operatorname{prod} \_\operatorname{exists}(\mathrm{P}, \mathrm{T}) \leftarrow$ new_prod$(\mathrm{Z}, \mathrm{T}), \operatorname{prod}(\mathrm{Z}: \mathrm{P}), \operatorname{product}(\mathrm{P}, \mathrm{T}-1)$.

event new_cust (cusino: integer; type: (w,r ); derived cust: CUSTOMER).

event new_store (stono: integer; derived sto: STORE).

event set_cost (prodno: integer; cost: integer; derived prod: PRODUCT). constraints

prod_not_exists $(P, T) \leftarrow$ set_cost $(Z, T)$, prod(Z:P), not product(P,T-1).

event replenishment (prodno: integer; stono: integer; qty: integer;

derived prod: PRODUCT; ps : PROD_STO).

event sale (prodno: inleger; stono: integer; custno: integer; qty: integer;

derived prod: PRODUCT; ps: PROD_STO;

cust: CUSTOMER; amount: integer).

aunount $(Z: A) \leftarrow \operatorname{prod}(Z: P), \operatorname{prodcos}(P, I-1: C o), q t y(Z: Q), A=Q^{*} \mathrm{Co}$

event payment(custno: integer; anount: integer; derived cust: CUSTOMER).

Figure 1. External events in the ODCM example 
The deduction rule of attribute amount of salc events, defines that the amount of a sale $\mathrm{z}$ is $a$ (amount(z:a)) if the prod attribute of $z$ is $p$ (prod(z:p)), the prodcost attribute of PRODUCT $p$ at time $\mathrm{t}-1$ is $c o$ (prodcost $(p, t-1: c o))$, the qly attribute of $\mathrm{z}$ is $q$ (qty(z:q)) and $a=T^{*} c o$.

Note that literals in deduction rules may be events (like new_prod(Z,T)), event attributes (like prod(Z:P)), objects (like product( $P, T-1)$ ), object altributes (like prodcost(P,T-1:Co)), and evaluable literals (like $A=Q{ }^{*}$ Co).

\subsection{Object Classes}

The definition of an object class C consists of:

- The existence rule, which is a deduction rule that states the conditions that an object must satisfy to be a member of the class at a given time.

- The attributes of the objects. For each attribute, we deline its name, domain and one or more deduction rules, which define the value of the altribute at a given time.

- The (static and dynanic) constraints of the objects. For each constraint, we define its nane and a deduction rule.

- The events generated by the objects. For each generded event, we define its name, the event attributes and one or more deduction rules, defining when the event is generated and the value of its atuributes.

We again give additional details using the example of Figure 2. We assume that each object has a unique identifier, which is usually given by the external events. A fact such as product(p,t) is true if $p$ is an identifier of an object of class PRODUCT at time $t$. A fact such as prodcosi $(\mathrm{p}, \mathrm{t}: \mathrm{co})$ is true, where $\mathrm{p}$ is an identificr of a product, if the prodcost aturibute of $p$ has value co at time $t$.

The existence rule of class PRODUCT defines that a product $p$ belongs to this class at time $t$ if an event new_prod occurred at a time $11(11 \leq t)$, such that its prod attribute has value $p$. Existence rules for STORE and C.USTOMER are quite similar. Class PROD_STO corresponds to the aggregation of a PRODUCT and a STORE. In the example, it is assumed that all products are present in all stores: this is what defines the existence rule for PROD_STO. Note the use of evaluable prodicale id(P,S,Ps) which relates the identifiers of PROD_STO to the product and store identiliers. If, for example, the identifier of a product_store is formed by the concatenation of the product and store identifiers then id(p1,sI,p1s1) could be a fact of this predicate. Nolc, however, that we do not need to further specify this predicate at the conceptual level. The existence rules for WHOLESALER and RETAILER define these classes as subsets of CUSTOMER.

Instances of the PRODUCT class have three altributes. To save space, we only give the domain (integer) of the first (produumber). The deduction rules give the definitions of these attributes. For instance, the goh_value of a product $p$ at time $t$ is defined as the sum of the values at time $l$ of this product in all stores. We assume that deduction rules are defined such that attributes are single-valued. 


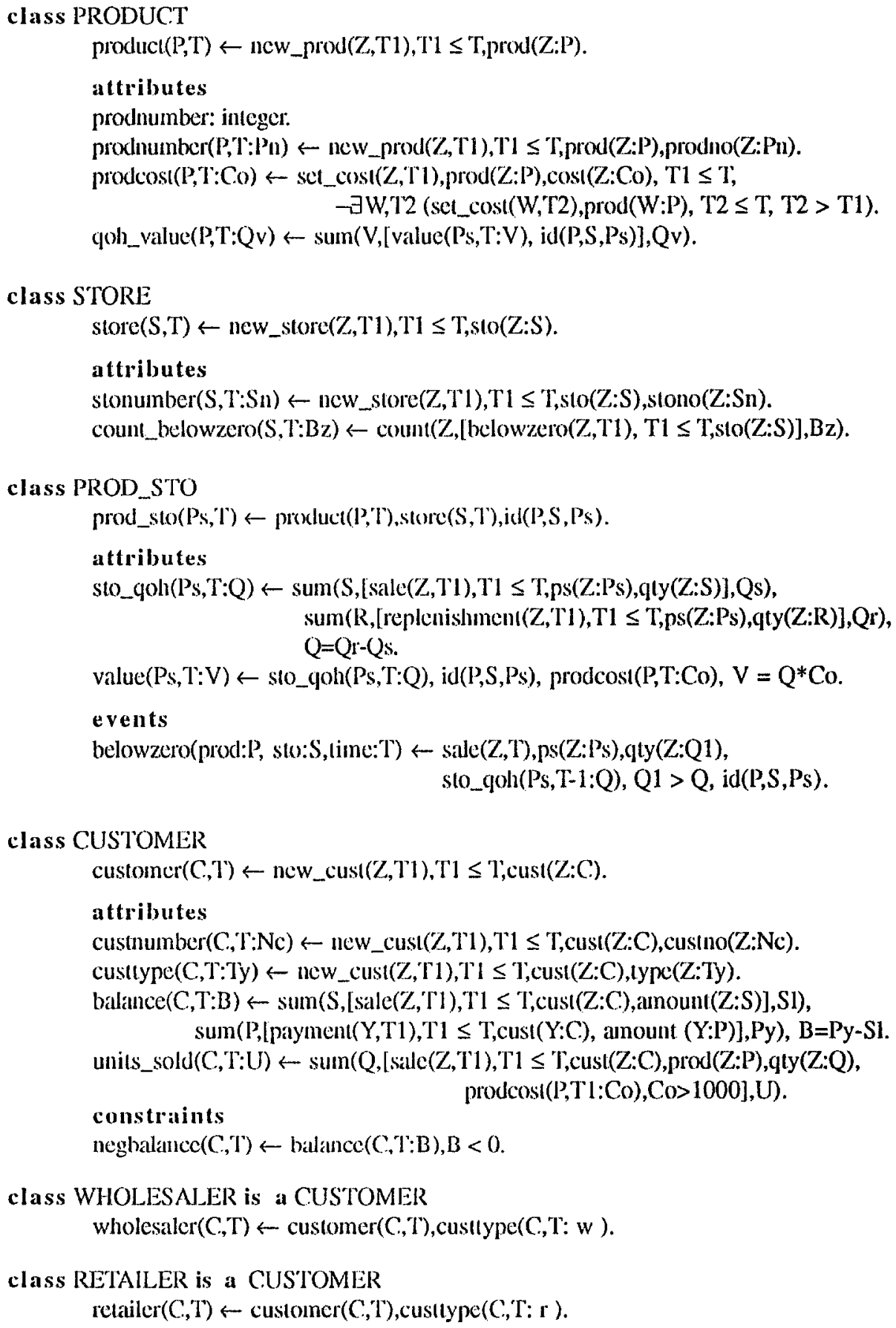

ligure 2. Object classes in the ODCM example 
Instances of the WHOLESALER and RETAILER classes inherit the attributes from CUSTOMER class.

There is a constraint associated with object class CUSTOMER. The name of the constraint is negbalance, which is a predicate name. A fact negbalance(c,l) is true, that is, the constraint is violated, if the balance of customer $\mathrm{c}$ is negative at time $\mathrm{t}$. It is assumed that the external events (a sale in this example) that induce a violation of a constraint will be rejectcd.

There is a generaled evcnt associated with object class PROD_STO. The name of the event is belowzero, which is a preclicate name, with three altributes. A fact belowzero(p,s,t) is true, that is, the event is generated, if the gly of a sale is greater than the sto_qoh at time t1. Like external events, generated events have also an internal identifier, which is given automatically by the system when they are generated. Note that in class STORE an attribute is defined (count_belowzero) that gives the count of such events generated up to a given time for a given store.

\section{Internal Events and Internal Events Model}

In this section, we briefly review the key concepts of internal events and internal events model. See [Oli89,San90] for further details. In the next section we will use the internal events model as a basis for determining the object interactions involved in an ODCM.

\subsection{Internal Events}

There are three kinds of internal events:

1) insertion, which capture the notion of inserting a new object into a class or giving an initial value 10 an object altribule.

2) delction, which correspond (1) deleting an object from a class or removing an aturibute value of an object.

3) modification, which capture the notion of changing an attribute value.

For each object class obj we define an insertion and a deletion internal event predicate as follows:

$$
\begin{aligned}
& \forall X, T[\operatorname{cobj}(X, T) \leftrightarrow o b j(X, T) \wedge \neg o b j(X, T-1)] \\
& \forall X, T[\delta o b j(X, T) \leftrightarrow o b j(X, T-1) \wedge \neg o b j(X, T)]
\end{aligned}
$$

Thus, for example, tproduct $(x, 1)$ is true if object $x$ is a member of class PRODUCT at time $\mathrm{t}$, and it was not a member of that class at time $\mathrm{t}-1$.

For each attribute alt we define an insertion, a deletion and a modification internal event as follows:

$$
\begin{aligned}
& \forall \mathrm{X}, \mathrm{T} \quad[\operatorname{lalu}(\mathrm{X}, \mathrm{T}: \mathrm{V}) \leftrightarrow \operatorname{aut}(\mathrm{X}, \mathrm{T}: \mathrm{V}) \wedge-\exists \mathrm{V} 1 \text { aut }(\mathrm{X}, \mathrm{T}-1: \mathrm{V} 1)] \\
& \forall X, T[\delta a t t(X, T: V) \leftrightarrow \operatorname{atu}(X, T-1: V) \wedge \neg \exists V 1 \text { att(X,T:V1)] } \\
& \forall X, T[\mu a t t(X, T: V, V 1) \leftrightarrow \operatorname{att}(X, T-1: V) \wedge \operatorname{att}(X, T: V 1) \wedge V \neq V 1]
\end{aligned}
$$


Thus, for example, $\mu$ sto_qoh(ps, $1: y b, 4 a)$ is true if the value for the attribute sto_qoh of product_store ps was $q b$ at time $1-1$ and is ga at lime 1.

For notational convenience, we also associale an insertion internal event predicate to each external, constraint or generated event. Thus, $I$ salc $(z, 1)$ will be the internal event associated to $\operatorname{salc}(z, 1)$, and tprod_exists $(p, 1)$ will be the internal event associated to constraint prod_exists of event new_prod.

From the above definitions, we have the following transition axioms:

$$
\begin{aligned}
& \forall X, T[\operatorname{obj}(X, T) \leftrightarrow(\operatorname{obj}(X, T-1) \wedge \neg \delta o b j(X, T)) \vee \operatorname{cobj}(X, T)]
\end{aligned}
$$

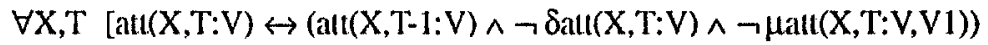

$$
\begin{aligned}
& \vee \operatorname{tatt}(X, T: V) \vee \mu a t t(X, T: V 1, V)]
\end{aligned}
$$

which define object existence or attribute values at time 1 in terms of object existence or attributc values at time $1-1$, and the internal events induced during a transition from $t-1$ to $t$.

\subsection{Internal Events Model}

From the definitions (1) - (5) given above, and using the transition axioms (6) and (7), we get the internal events model corresponding to a given ODCM. The internal events model consists of a set of rules. Each rule delines the condition for the occurrence of an internal event. We reler again to [Oli89.San90)] for details on the procedure that derives automatically the intcrial cvents model.

Figure 3 shows part of the internal events model corresponding to the ODCM example. In the following, we comment the internal events rules related with object class PROD_STO. The first two rules define the conditions for the insertion of a product_store ps in its class, which are:

1) An internal event $t$ product $(p, t)$ is induced in a transition, a store $s$ exists at time $t$ and the identilier of the new object is $\mathrm{ps}$, or

2) An internal event tstore( $s, 1)$ is induced in a transition, a product p exists at time t and the identificr of the new object is ps.

The next five rules define insertions and modifications of attributes sto_qoh and value. Note, from the ODCM definition, that such attributes values are never deleted, since object instances of the classes in our example are never deleted.

The last rule defines the condition for the generation of internal event tbelowzero: when an $\mathrm{t}$ sale occurs at time $\mathrm{T}$ with a qly greater than sto_goh at time T-1. 
(in class STORE)

rs.1 istore(S,T) $\leftarrow$ inew_store $(Z, \Gamma)$, sto $(Z: S)$.

(attributes)

rs.2 tstonumber(S,T:Sn) $\leftarrow$ inew_store $(Z, T)$,sto(Z:S),stono(Z:Sn).

rs.3 tcount_belowzero(S,T:0) $\leftarrow$ istore $(S, T)$.

rs.4 $\mu$ count_belowzero(S,T:C.b,Ca) $\leftarrow$ count_belowzero(S,T-1:Cb), count(Z,[tbelowzcro(Z,T),sto(Z:S)],C), $\mathrm{Ca}=\mathrm{Cb}+\mathrm{C}$.

(in class PROD_STO)

rps.1 tprod_sto(Ps,T) $\leftarrow$ iproduct(P,T),siore(S,T),id(P,S,Ps).

rps.2 tprod_slo(Ps, I) $\leftarrow$ product $(P, T)$, istore(S,T),id(P,S,Ps).

(attributes)

rps.3 tsto_qoh(Ps,T:0) $\leftarrow$ iprod_sto(Ps,T).

rps.4 $\mu s t o \_q o h(P s, T: Q b, Q a) \leftarrow \operatorname{sum}(S,[t \operatorname{sale}(Z, T), p s(Z ; P s), q t y(Z: S)], Q s)$, $\operatorname{sum}(\mathrm{R},[$ r replenishment( $\mathrm{Z}, \mathrm{T}), \mathrm{ps}(\mathrm{Z}: \mathrm{Ps}), \mathrm{qty}(\mathrm{Z}: \mathrm{R})], \mathrm{Qr})$, sto_qoh(Ps,T-1:Qb), Qa=Qb+Qr-Qs, Qa $\neq$ Qb.

rps.5 ivalue(Ps,T:0) $\leftarrow$ tprod_sto(Ps,T).

rps.6 $\mu$ value(Ps, T:Vb,Va) $\leftarrow \mu$ sio_qoh(Ps,T:Qb,Q), id(P,S,Ps), prodcosi $(\mathrm{P}, \mathrm{T}: \mathrm{Co})$, valuc(Ps, T-1:Vb), Va $=\mathrm{Q}^{*} \mathrm{Co}$.

rps.7 $\mu$ value(Ps,T:Vb,Va) $\leftarrow$ sio_qoh(Ps,T:Q), id(P,S,Ps), $\mu$ prodcosi(P,T:Cob,Coa), value(Ps, T-1:Vb), Va = Q*Coa.

(generated events)

rps.8 tbelowzero(prod:P, sto:S,limc:T) $\leftarrow$ lsale(Z,T),ps(Z:Ps),qty(Z:Q1), sto_qoh(Ps, $\mathrm{T}-1: \mathrm{Q}), \mathrm{Q} 1>\mathrm{Q}$,id(P,S,Ps).

Figure 3. Part of the internal events model of the ODCM example

\section{Object Interaction in an ODCM}

In this section, we outline a formal procedure that determines the object interactions implied by a given ODCM.

\subsection{Target Events}

We define as target events of an object class those internal events that must be produced by the object class or some of its instances in order to fulfil their task. More specilically, the larget events are: 
1) The insertion and deletion events of the objects. An object class must be able to create object instances and, thus, producing the corresponding insertion cvents. To each creation of an object instance corresponds an insertion event. An object instance must be able to remove itsclf from its class, producing the corresponding deletion event.

2) The insertion, deletion and modification of object attribute values. An object instance must be able to inilialise, remove or chenge the value of its attributes producing the corresponding insertion, deletion or modilication events.

3) The insertion event of constraints. An object instance or class must be able to produce such events. If they are indeed produced in a transition the current transaction must be rejected.

4) The insertion events of generated events. An object instance or class must be able to produce such events when the corresponding conditions are satisfied.

The target events can be casily determined from the internal events inodel: they correspond to the predicates in the head of some rule. In the example of figure 3 , the target events of object class PROD_STO are: Iprod_sio, isto_qoh, $\mu$ sio_qoh, ivalue, $\mu$ value and tbelowzero.

\subsection{Relevant Events}

We define relevant events for an object class as those events that must be communicated to the object class or to some of its instances in order for them to be able to produce the target events. Relevant events are those that appear in the body of some rule of the internal events model.

In the example of ligure 3, relevant events lor object class PROD_STO are: tproduct, istore, tprod_sto, tsale, treplenishment, $\mu$ sto_goh and $\mu$ prodcost. Note that the same internal event may be both target and relevant for a class. In the example, $\mu$ sto_qoh is both a targct event (nccessary for maintaining the value of altribute sto_qoh) and a relevant event (must be commuticated in order to produce $\mu$ value).

\subsection{Induction of Events}

Rules in the internal events model establish a relationship between relevant and target events: target events appear in the head of the rule, and relevant events appear in its body. We call induces to this relationship. Thus, we say induces $(a, b)$ if $a$ is a relevant event for $b$. Occurrence of internal cvent a may induce the occurrence of internal event $b$. Note that this is a potential induction, since actual induction depends on other conditions.

Thus, if we consider the rule:

$$
\begin{aligned}
\text { t belowzero(prod:P, sto:S, time:T) } & \leftarrow \text { tsale(Z,T),ps(Z:Ps),qty(Z:Q1), } \\
& \text { sto_qoh(Ps, T-1:Q),Q1 > Q, id(P,S,Ps). }
\end{aligned}
$$

we find induces ( $\mathrm{t}$ sale, $\mathrm{t}$ belowzero). However, occurrence of a particular $\mathrm{tsale}$ may actually induce or not an tbelowzero internal event. 
The set of "induces" facts that corresponds to figure 3 are:

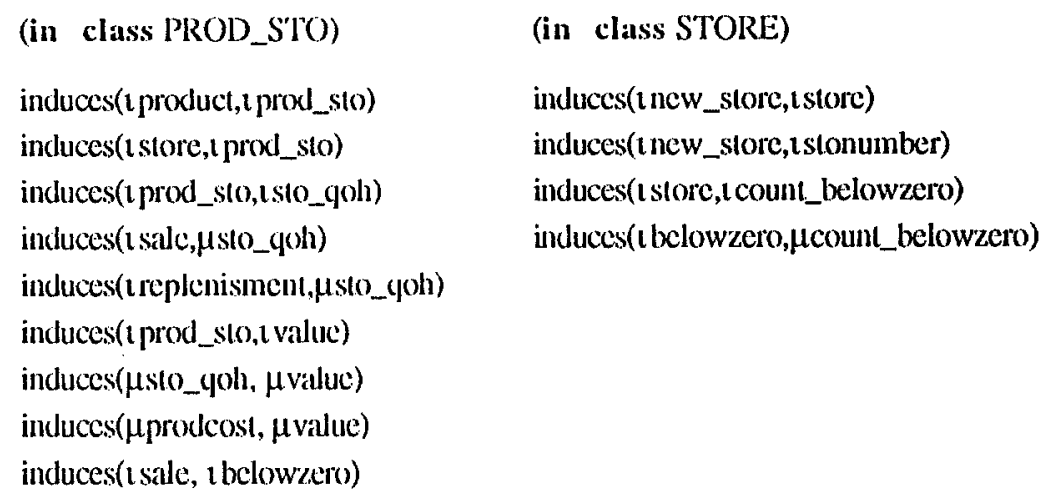

Knowing that a particular cvent $e$ has been induced, the rules:

$$
\begin{aligned}
& \text { induced }(X) \leftarrow \text { induces }(Y, X) \text {, induced }(Y) \text {. } \\
& \text { induced }(e) \text {. }
\end{aligned}
$$

compute the events induced by $e$. In the example above, if we know that event tnew_store has been induced, the rules give the events induced by it: tncw_store, tstore, tstonumber, tcount_below\%cro, tprod_sto, tsto_qoh and i value.

\subsection{Object Interaction Model}

Several object interaction models can be built for a particular system. We discuss some of them here, but others are possible.

In the first model, a central component, that we call the Events Manager, is responsible of all interactions. Its functions are:

1) To receive all extcrnal events produced at a given time. We allow the possibility of receiving several simultancous events from the environment.

2) To send to event classes the external events just received, in order to check that they salisfy the constraints and to compute the derived altributes defined at those event classes.

3) To send to object classes relevant events for the production of new object instances, and to receive the insertion events that may be produced by them.

4) To send to object instances relevant events for the destruction of object instances or updating object attributes, and to receive the events that may be produced by them.

5) To send to object classes or instances relevant events for constraints checking or the generation of events, and to receive the events that may be produced by them. 
In this model, a method must be detined lor each relevant cvent of an object and event class. The functions of these inethods are:

1) To receive the relevant event.

2) To produce the target events directly induced by the received event, thus creating or destroying object instances, updating object attributes, checking integrity constraints or generating events.

3) To send back to the Events Manager the set of target events produced.

We assume that other methods exist for accessing and returning attribute values. We will not consider them in our object interaction analysis, since they do not pose significant problems.

As an illustration of this model, we give below (and show in figure 4) an object interaction pattern that would evolve in the cxample of figure 3 , when the external event tnew_store occurs:

1) The event tnew_store is received by the Events Manager.

2) The Events Manager sends the event tnew_store to a method of event class new_store. This method would check if the event satisfies the constraint store_exists (similar to prod_existx) and would computed attribute sto. For the sake of simplicity, in this paper we will not describe any further this kind of interaction between the Events Manager and the event classes.

3) The Events Manager sends the event tnew_store to method MS1 of class STORE. The method adds a new instance to class STORE and initialises attribute stonumber to 0 , and returns events istore and $t$ stonumber:

4) The Events Manager sends the received event istore to method MS2 of class STORE. The method initialises attribute count_belowzero to 0 , and returns tcount_belowzero.

5) The Events Manager sends the event istore to method MPS1 of class PROD_STO. The method adds a new instance to class PROD_STO for each existing product instance, and returns the cvents tprod_sto thus produced.

6) The Events Manager sends each tprod_sto event received to method MPS2 of class PROD_STO. The method initialises attributes sto_qoh and value to 0 , and returns the events $\mathrm{isto}$ qoh and $i$ value.

It is interesting to note that the above object interaction pattern is just one of the many valid patlens. (Jher patterns would produce the same overall effect. 


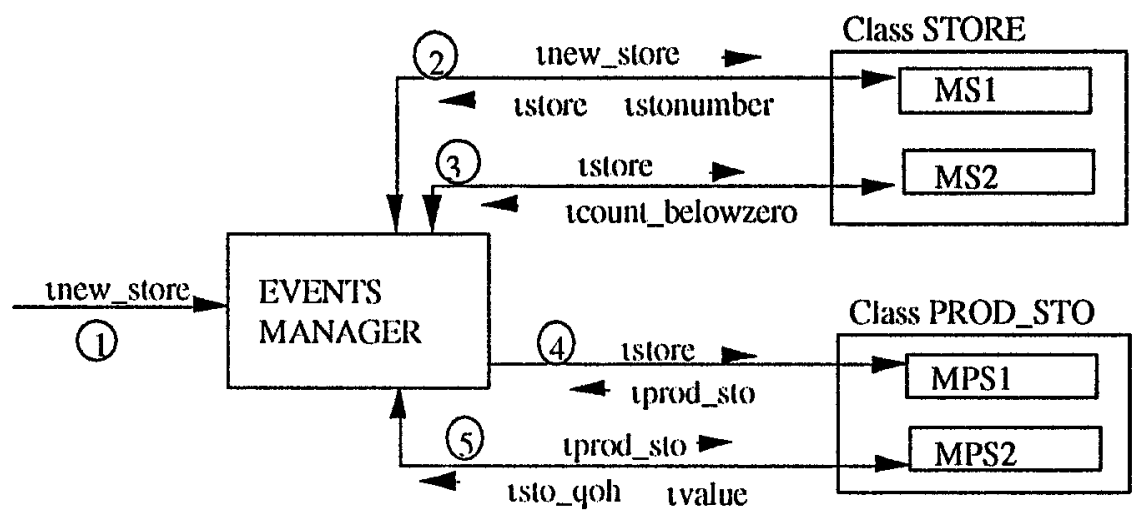

Figure 4. Example of object interaction pattern

In an alternative object interaction model, methods would produce all target events induced in a class, either directly or indireclly, by the received event. This model would reduce the need for object interaction, but then the methods become larger and less robust to changes (for instance, addition of a new object attribute might require the modification of existing methods). In some way, this is used by Oblog and TROLL ([SFS+89,SRG+91, JSH+91]).

Another object interaction model would eliminate the need of a central Events Manager, by allowing direct interaction between objects. However, methods then become still larger and even less robust to changes. Addition of new object classes, for instance, might require changing existing methods to reflect the need for interaction with the new objects. This model is used by most methods (see, for instance [Bai89,CoY91, Wie91,Prak92]).

In what follows, we outline a procedure for delermining object interaction patterns according to the first model, but we could adapt it to other models (see [Que92]).

\subsection{Valid Object Interaction Patterns}

In our model, we call object interaction paltern (shorlly, interaction) a sequence of messages sent by the Events Manager to methods in object classes, in response to one or more extcrnal events received from the environment.

We describe the sending of a message to a method of an object class by means of a predicate send(seqnumber,message,class, method), where the lirst argument is a sequence number. In this way, the interaction described above, for the external event tnew_store, would be defined by the sequence: 
send(1,1new_store,class_STORE,melhod_MS1)

$\operatorname{send}(2,1$ store,class_STORE,method_MS2)

$\operatorname{send}(3$, , store,class__PROD_STO,method_MPS1)

send(4,1prod_sto,class_PROD_STO,method_MPS2)

The sequence number will also be used to distinguish between states in an interaction. The final state of an interaction will be the greatest sequence number ( 4 in the example above).

We can also define the simulaunous sending of one or more events to different methods. In this case, we would just assign the sime sequence number to two or more "send" facts.

Before describing how such object interaction patlerns can be generated, we consider the issue of validity of a given interaction. To this end, we need to introduce first the concept of "computed".

Rules in the internal cvents model state the events that must be sent to a class to compute a given target event. In gencral, however, a target event can have more than one rule (for instance, tprod_sto has two rules: rps.1, rps.2).

A target event e of class $c$ delined by a rulc $r$ with relevant event $e_{1}$, is partially computed at some slate $S$ (denoled by peompuled $(S, \mathrm{c}, \mathrm{r})$ ) if:

a) $e_{1}$ has not been induced, or

b) $\mathrm{e}_{1}$ has been induced and it has been sent at some state $S 1$ to the corresponding method $\mathrm{m}$ (send $\left(\mathrm{S} 1, \mathrm{c}_{1}, \mathrm{c}, \mathrm{m}\right)$ ), with $\mathrm{S} 1 \leq \mathrm{S}$.

This definition can be extended casily with more than one relevant event (see example below).

A target event e is computed at some state S (denoted computed(S,e)) if it has been partially computed with respect to all its rules:

compuled $(S, \mathrm{e}) \leftarrow \operatorname{pcomputed}\left(S, \mathrm{c}, \mathrm{r}_{1}\right), \ldots ., \mathrm{pcomputed}\left(\mathrm{S}, \mathrm{e}, \mathrm{r}_{\mathrm{i}}\right)$.

The following rules deline the "compuled" and "pcomputed" predicates for the target event Iprod_sto of class PROD_STO:

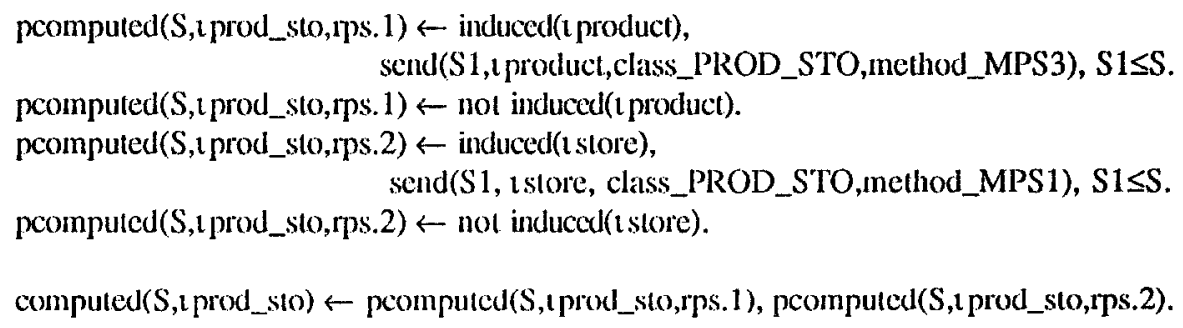


We also define the "computed" concept for the existence of object instances. We say that compuled(s,object_class) is true if the insertion and deletion internal events corresponding to the creation and destruction of the object instances have been computed at state $\mathbf{s}$. Formally, if oc is an object class:

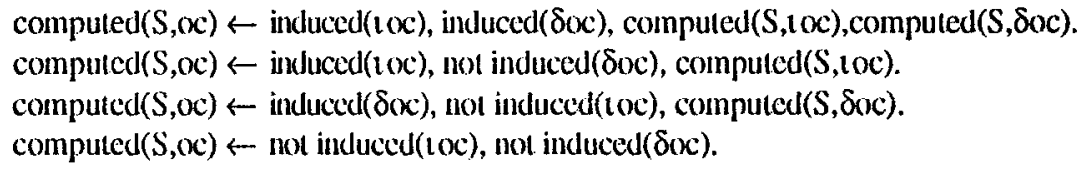

Note that the last rule defines that the object instances of oc are computed at any state of a transition if the roc and $\delta o c$ have not been induced, since in such case the extension of oc is not changed.

The following rules define the "computed" and "pcomputed" predicates for the target event $\mu$ sto_qoh of class PROD_STO:

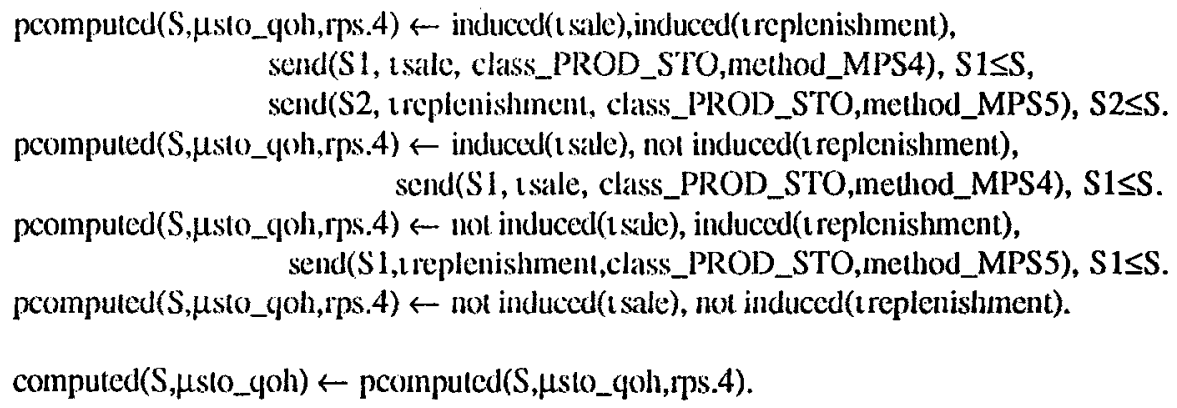

We also define the "computed" predicatc for attributes as follows:

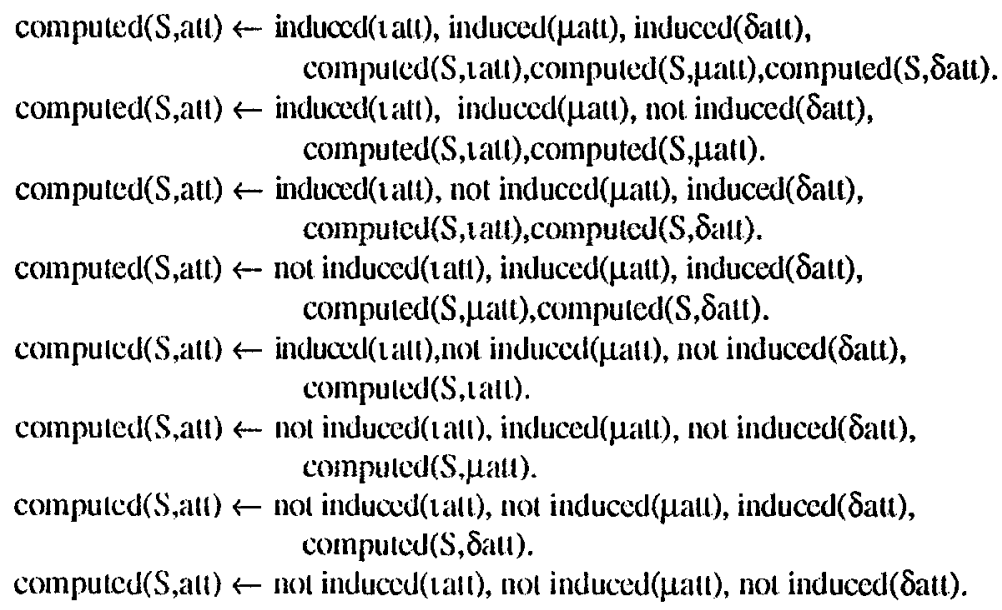


In principle we could define the validity of an object interaction pattern I for a set $E$ of extemal events, as:

$$
\forall X \text { (induced }(X) \rightarrow \text { compuled(fs, } X))
$$

where $\mathrm{fs}$ is the final statc, meaning that $\mathrm{I}$ is valid for $\mathrm{E}$ if all induced target events are computed by 1 . However, this does not take into account the interaction constraints that I must satisfy. These constraints, that can also be obtained from the internal events model, state the conditions that must hold for sending a message to a method.

For example, the following rules deline the interaction constraints associated to the relevant events of target event iprod_sto of class PROD_STO:

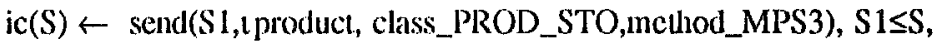

$$
\begin{aligned}
& \mathrm{S} 2=\mathrm{S} 1-1 \text {, nol computed(S2,store). } \\
& \text { ic(S) } \leftarrow \operatorname{send}(S 1, \text { istore, class_PROD_STO,method_MPS1), S1 } \leq S \text {, } \\
& \text { S2 = \$1 - 1, not compuled (\$2,product). }
\end{aligned}
$$

First constratint is violated if t product is sent to method MPS3 of class PROD_STO at some state $S 1$, and store object instances had not been compuled in the previous state. This is so because MPS3 requires lo know which stores exist before creating new product_store instances. Recall that we allow the creation of both products and stores in the same transition (that is, we allow that one or more tnew_prod and tnew_store external events occur simultaneously). Second constraint is similar.

Having defined the "induced" and "computed" concepts, and the constraints that an interaction must to fullil, we say that an object interaction pattern I is valid for a set of external events $E$ if all events induced by $E$ are computed by I and no one ic is violated.

$$
\forall X \text { (induced }(X) \rightarrow \operatorname{computed}(\text { Is, } X), \text { not ic }(f s))
$$

\subsection{Generation of Object Interaction l'atterns}

Generation of object interaction patterns consists in obtaining the messages to be sent by the Events Manager to methods in object classes, in response to the external events received at a given time by the system. The interaction generated is different depending on the external events received.

We outline in this section our procedure for the automatic generation of interaction, which is based on plan generation techniques.

Let $E$ be a set of external events, that the Events Manager can receive at the same point of lime. If we know $E$ in advance the interaction can be generated at development-time. On the contrary, it must be generaled by the Events Manager at execution-time. 
The procedure uses the computed, pcomputed and ic predicates and their definition, presented in last scction. Recall that these rules can be derived automatically from the internal events model.

Lel us denote by $U$ the set of events induced by $\mathrm{E}$.

Let $C$ be the set of target events already compuled at some stage of our procedure. At the beginning, C consists of the elements of set E. Let I be the set of messages to be sent by the Events Manager. Initially, $I=\varnothing$. Our procedure consists of 6 steps:

1) Select an element elem firom sel C.

2) Sclect a set $R$ of rules having a send(S, elem,class,method) literal in their body with the same class and method.

3) Extract from set $R$ the subset $Q$ of relevant rules. A rule in $R$ is relevant if:

- for all its literals induced(elemI) appcaring in its body, eleml is in U.

- for all its literals not induced(eleml) appearing in its body,eleml is not in U.

4) Check that all rules in sel $Q$ are applicable. All rules in $Q$ are applicable if there is not any constraint violated by $I \cup$ send(S,elem,class,method).

5) If all rules in $Q$ are applicable, then:

- Add the send(S, elem,class,method) literal to I. Assign to the sequence number of this newly added "send" literal the current state. Add 1 to the current statc.

- Add to $\mathrm{C}$. the elem2 apparing as pcompuled(S, elem2, $\mathrm{R})$ in the heads of each rulc $\mathrm{Q}$, only if elem2 is totally computed at the current state.

6) The procedure linishes as scon as $U$ is a subset of C.

We illustrate the procedure by means of an example. Assume the set of external events received by the Events Manager is $\mathrm{E}=\{\mathrm{t}$ sale, $t$ sel_cosi $\}$.

One solution of the generation procedure of object interaction patterns is:

$$
\begin{aligned}
& I=(\text { send(1,1sale,PROD_STO,method_MPS4), } \\
& \text { send(2, iset_cost,PRODUC.T,method_MP2), } \\
& \text { send(3, tsale,C.USTOMER,method_MC.3), } \\
& \text { send(4, } \mu \text { sto_qol,PROD_STO,method_MPS6), } \\
& \text { send(5, } \mu \text { value,PRODUCT,method_MP3), } \\
& \text { send }(6, \mu \text { balance,CUSTOMER,method_MC4), } \\
& \text { send }(7, \text { t belowzcro,STORE, method_MS3)\} }
\end{aligned}
$$

Fig. 5 shows graphically and explain the messages sent by the Events Manager to the objects. 


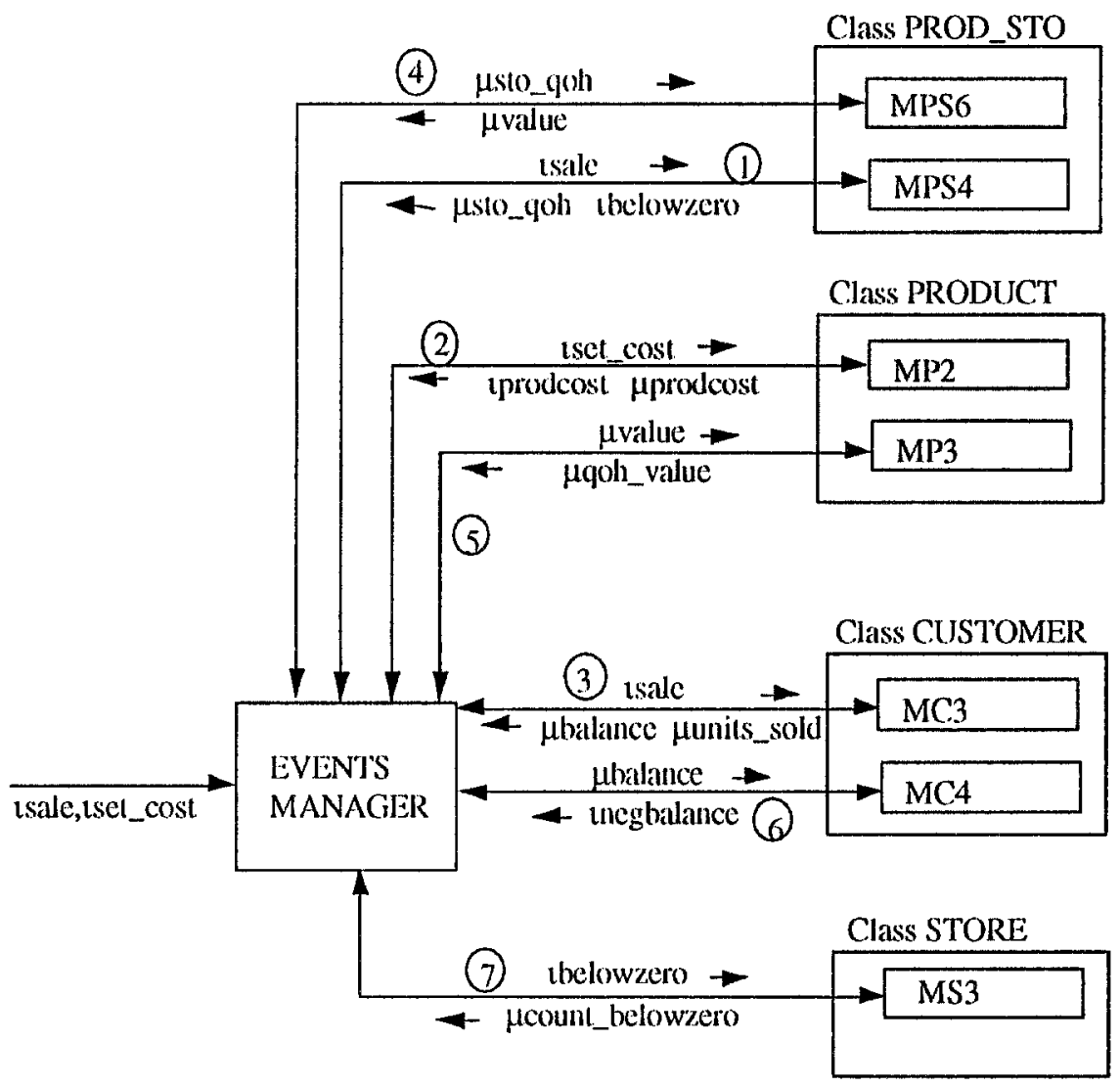

1) The Events Manager sends the event tsale to method MPS4 of class PROD_STO. The method modifies the sto_yoh value, and produce the generated event thelowzero, that is relurned with the event $\mu$ sto_goh.

2) The Events Manager sends the event tset_cost to method MP2 of class PRODUCT. The method initialises or modify the value of attribute prodcost, and returns the events tprodcost, $\mu$ prodcost.

3) The Events Manager sends the event tsale to method MC3 of class CUSTOMER. The method modifies the values of attributes balance and units_sold, and returns the events $\mu$ balance, $\mu$ units_sold.

4) The Events Manager sends the event $\mu$ sto_qoh to method MPS6 of class PROD_STO. The method modifies the value of attribute value, and returns the event $\mu$ value.

5) The Events Manager sends the event $\mu$ value to method MP3 of class PRODUCT. The method modifies the value of attribute qoh_valuc. and returns the event $\mu$ goh_value.

6) The Events Manager sends the event phalance to method MC4 of class CUSTOMLR. The method checks the negbalance constraint, and returns tnegbalance if violated.

7) The Events Manager sends the event tbelowzero to method MS3 of class STORE. The method modifies the value of altribute count_belowzero, and returns the event $\mu$ count_belowzero.

Figure 5. Object Interaction pattern example 
We now explain in detail how the solution is obtained. The events induced by $\mathrm{E}$ are:

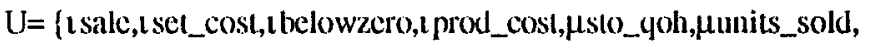

$$
\begin{aligned}
& \mu \text { balance, } \mu \text { value, }, \imath \text { negbalance, } \mu \text { prodcost }, \mu \text { count_belowzero, } \mu \text { qoh_value\} }
\end{aligned}
$$

The set of computed target events, and the set of send literals, at the beginning of the process are:

$$
\begin{aligned}
& C_{0}=\{\text { isalc, } t \text { set_cost }\} \\
& I_{0}=\{\}
\end{aligned}
$$

1) We select an element of $\mathrm{C}_{0}, \quad$ elem $=$ tsale.

2) We select the set R from rules associated to target cvents of class PROD_STO:

$$
\begin{aligned}
& \text { pcompuled( }\left(S, \mu s t o \_ \text {_oh.rps.4) } 4\right. \text { induced(tsule), not induced(treplenishment), } \\
& \text { send(SI, tsale, class_PROD_STO,method_MPS4), S1 } \leq S \text {. } \\
& \text { pcomputed(S.Hsto_qoh.rps.4) } \leftarrow \text { induced(tsale), induced(treplenishment), } \\
& \text { send(SI. tsale. class_PROD_SlO.method_MPS4), SISS, S2sS. } \\
& \text { send(S2. treplenishment, class_PROD_STO,method_MPS5). } \\
& \text { pcomputed(S, thelowzero,rps.8) } \leftarrow \text { induced(tsale) } \\
& \text { send(S1,tsale,class_PROD_SIO.method_MPS4), SISS. }
\end{aligned}
$$

3) We extract from $R$ the subsel of relevant rules

$$
Q=\{\text { C.R2,CR.5 }\}
$$

4) We check if rules in $Q$ are applicable. They are applicable because there is not any ic violated by $I_{0} \cup$ scid(S, isale, class_PROD_STO,method_MPS4).

5) We add send(S, isalc, class_PROD_STO,method_MPS4) literal to $I_{0}$, assigning the current state to the sequence number of this literal:

$$
I_{1}=\{\text { send(1,isale,PROD_STO,incthod_MPS4) }\}
$$

We add to $C_{0}$ the events that will be totally computed once the tsale message has been sent to method MPS4 of class PROD_STO. We know which are these events by the following computed rules:

$$
\begin{aligned}
& \text { computad }(S, \mu \text { sto_goh }) \leftarrow \operatorname{pcomputed}(S, \mu \text { sto_goh,rps.4) } \\
& \text { computcd }(S, t \text { belowzcro }) \leftarrow \operatorname{pcomputed}(S, \text { ibclowzero,rps.8) }
\end{aligned}
$$

So, the new set of computed larget events is:

$$
C_{1}=\{\text { sale,tset_cost, } \mu \text { sto_goh,t belowzero }\}
$$

6) Given that $U$ is not a subset of $C$ yet, then it is necessary to repeat the process. 
We outline the next iterations in the following. Eight iterations are needed to arrive to the object interaction pattern example:

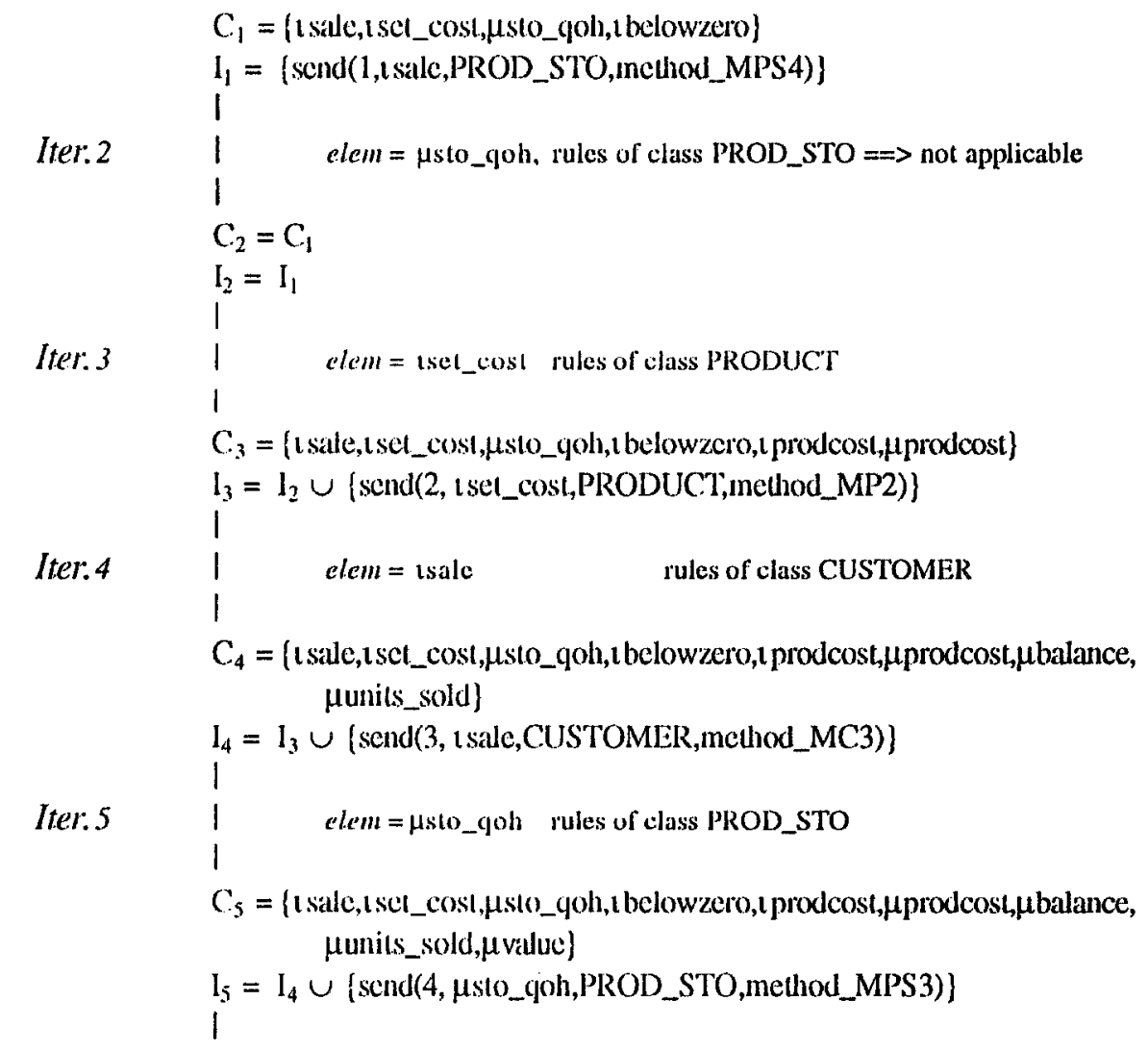
It. $6,7,8$

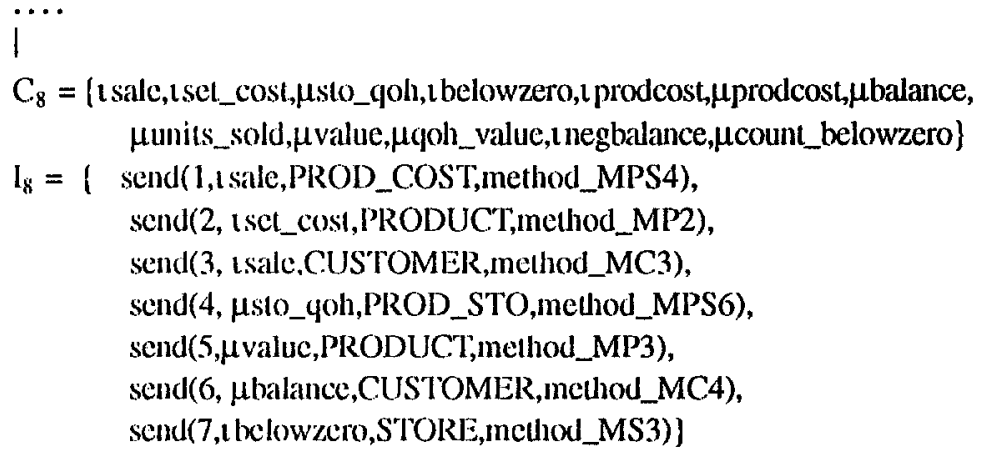

At the end of the 8 th iteration, we have in C. all the target events that can be induced (U is a subset of $\mathrm{C}$ ), and the procedure finishes. 
In all iterations the element selected from $C$ to be sent to a method of a class did not violated any interaction constraint, except in lier.2 where the purpose was to modify the value of the goh of products in a store, when the cost of the products was not already modilied (having a set_cost event in set E). In that case the following ic were violated by the selected event fisto_goh:

$$
\begin{aligned}
& \text { ic }(S) \leftarrow \text { send(S1, } \mu \text { sto_qoh, class_PROD_STO,method_MPS6), } S 1 \leq S \text {, } \\
& \text { S2 = S1 -1, not computcd(S2,prodcost). }
\end{aligned}
$$

There is more than one valid object interaction pattern for the same set of external events. We obtain different interactions depending on how the elements in set $\mathrm{C}$ are selected in step 1 of the procedure.

For example, another valid solution given by our procedure, would be:

$$
\begin{aligned}
I=\{ & \text { send(1, iset_cost,PRODUCT,method_MP2), } \\
& \text { send(2, isale,CUSTOMER,method_MC3), } \\
& \text { send(3, isale,PROD_STO,method_MPS4), } \\
& \text { send(4, } \mu \text { balance,CUSTOMER,method_MC.4), } \\
& \text { send(5, ibelowzero,STORE,method_MS3) } \\
& \text { send(6, } \mu \text { sto_qoh,PROD_STO,method_MPS6), } \\
& \text { send(7, } \mu \text { valuc,PRODUCT,method_MP3) }\}
\end{aligned}
$$

\section{Discussion}

The results from the previous section show that a procedure exists that determines the object interactions implied by a given ODCM. We can then pose the question whether explicit object interaction, as present in most current object-oriented methods, is a desirable feature for conceptual models of information systems. We believe that a definite answer to that question is not possible yet. However, we hope that the following comments might provide some elements for that answer (see also [HeE90,EFG+91, (1C.P92]).

Our procedure shows that object interaction relies on some object interaction model. We have seen in section 4.4 that several models are possible, and that object interactions are quite different from a model to another. On the other hand, changing from a model to another is a difficult task. Such a task might be necessary if the object interaction model used at the conceptual lcvel is different from the one used at the design level.

Our procedure also shows that several, cquivalent object interaction patterns may exist for the same input external events. Explicit object interaction modelling forces to choose arbitrarily one of them at the conceptual level. 
It can also be shown that object-oriented models using direct inter-object communication are very sensilive to changes. Additions of new object classes or attributes may imply changes in existing methods. In our example, assume the case when a single-instance object class COMPANY is added to the model, with aturibute global_qoh_value. Then, methods at the product_store or product level should be changed in the way that they now communicate to that new object the changes on the qoh_values.

Some object-oriented methods allow defining derived attributes, that is, attributes whose value may be computed from the values of other attributes of the same or different object. In these cases, the procedure for evaluating the attributes may have an impact on object interactions. If, at the design level, the value of the attribules is computed whenever they are requested, then no object interaction is necessary: the object method evaluates the defining rule and returns the result. However, if the value for that allribute is made explicit in the object local stale, then the need arises for new interaction: the object must know changes on the values of the terms in the defining rule.

\section{Conclusions}

We have described the main components of an object-oriented, deductive approach (ODCM) to conceptual modelling of information systems. Basic concepts of this approach are event and object classes. Event classes have attributes and constraints. Object classes have attributes, constraints and generaled events. Rules specified at the class level relate object existence, and atuibute values to events. Object interaction is not made explicit.

We have then presented a formal procedure, based on the concepts of internal event and internal events model, that produces the object interaction patterns implied by an ODCM. We have also formalized the concept of valid interaction.

We have discussed the issue of whether explicit object interaction is a desirable feature of conceptual models. Some (hopefully) relevant points to that issue have becn made, based on the above results.

We plan to implement the object interaction patterns generation procedure for several object interaction models, and the automatic generation of methods.

\section{Acknowledgements}

We would like to thank Dolors Costal, Carme Martin, Enric Mayol, Joan Antoni Pastor, Maria Ribera Sancho, Jaume Sistac, Ernest Tenicnte and Toni Urpí for many useful discussions. We are also grateful to the referees for their interesting comments and suggestions.

This work has been partially supported by the CICYT PRONTIC program project TIC 680. 


\section{References}

[Bai89] Bailin, S.C. "An object-oricnted requirements specification method", Comm. of the ACM, Vol.32, No.5, May 1989, pp. 608-623.

[CoY91] Coad,P.; Yourdon,E. “Object-oriented analysis”. Yourdon Press, 1991.

[dCP92] de Champeaux,D.; Faure,P. "A comparative study of object-oriented analysis methods". Jourual of Object-Oriented Programming, March-April 1992, pp.2123.

[EFG+91] Estier,Th.; Falyucl,G.; Guyol,J.; Leonard,M. "Six spaces for global information systems design". In [VMR91], pp.343-359.

[FaL89] Falkenberg,E.D.:Lindgreen,P. (Eds.). "Information system concepts: An in-depth analysis", North-Holland, 1989.

[GKB82] Gustafsson,M.R.;Karlsson,T:;Bubenko,J. "A declarative approach to conceptual information modcling". In Olle,T.W.; Sol,H.G.;Verrijn-Stuart,A.A. (Eds.) "Information systems design mcthodologies: A comparative review", NorthHolland, 1982, pp. 93-143.

[HeE90] Henderson-Sellers,B.;Edwards,J. "The object-oriented systems life cycle". Comm. of the ACM, Vol.33, No.9, Seplember 1990, pp. 143-159.

[JaR84] Jardine,D.A.;Rcuber,A.R. "Information scmantics and the conceptual schema". Information Systcins, Vol.9,No.2,1984, pp. 147-156.

[JSH+91] Jungclaus,R.; Saake,G.; Hartmann,T.; Scrnadas,C. "Object-Oriented Specification of Information Systems: The TROLL Language". Technische Universitäl Braunschwcig. Informatik-berichte, 91-04.

[MSS92] Mayol,E.; Sancho,M.R.; Sistac,J. "The ODISSEA projecl: an environment for development of Information Systems". Procecdings of the third International Workshop on the Deductive Approach to IS and DB. Roses. Sept, 1992. pp.127156.

[Oli86] Olivé, A. "A comparison of the operational and deductive approaches to conceptual information systems modelling". In "Information Processing 86", Elsevier, 1986, pp. 91-96.

[Oli89] Olivé, A. "On the design and implementation of information systems from deductive conceplual models". Proc. of the 15th. VLDB, pp. 3-11.

[Prak92] Prakash,N. "An object-oriented methodology for information systems design". In Falkenberg,E.D.,Rolland,C.,El-Sayed,E.N. (Eds). "Information system concepts: improving the understanding", North-Holland, 1992, pp.87-122.

[Que92] Quer,C. "Combining the object-oriented approach and the deductive approach for conceptual modelling". Proceedings of the third International Workshop on the Deductive Approach to IS and DB. Roses. Sept, 1992. pp.127-156. 
[San90] Sancho, M.R. "Deriving an internal events model from a deductive conceptual model". Proc. Intl. Workshop on the deductive approach to IS and DB, S'Agaró, 1990, pp. 73-92.

[SFS+89] Scrnadas, A.;Fiadeiro,I,SScrnadas,C.;Ehrich,H-D. "The basic building blocks of information systems". In [FaL89], pp. 225-246.

[SRG+91] Sernadas,C.:Resende,P.;Gouveia,P.;Sernadas,A. "In-the-large object-oriented design of information systems", in [VMR91], pp. 209,232.

[Wic91] Wieringa,R.J. "Object-oriented analysis, structured analysis and Jackson system development", in [VMR91], pp. 1-21.

[VMR91] Van Assche,F.;Moulin,B.;Rolland,C.. (Eds.) "Object oriented approach in information systems". North-Holland, 1991. 